\title{
Notes on the zero-divisor graph and annihilating-ideal graph of a reduced ring
}

\author{
Mehdi Badie
}

\begin{abstract}
We translate some graph properties of $\mathbb{A} \mathbb{G}(R)$ and $\Gamma(R)$ to some topological properties of Zariski topology. We prove that the facts "(1) The zero ideal of $R$ is an anti fixed-place ideal. (2) $\operatorname{Min}(R)$ does not have any isolated point. (3) $\operatorname{Rad}(\mathbb{A} \mathbb{G}(R))=3 . \quad(4) \operatorname{Rad}(\Gamma(R))=3$. (5) $\Gamma(R)$ is triangulated (6) $\mathbb{A} \mathbb{G}(R)$ is triangulated." are equivalent. Also, we show that if the zero ideal of a ring $R$ is a fixed-place ideal, then $\operatorname{dt}_{t}(\mathbb{A} G(R))=|\mathcal{B}(R)|$ and also if in addition $|\operatorname{Min}(R)|>2$, then $\operatorname{dt}(\mathbb{A} \mathbb{G}(R))=|\mathcal{B}(R)|$. Finally, it is shown that $\operatorname{dt}(\mathbb{A} \mathbb{G}(R))$ is finite if and only if $\mathrm{dt}_{t}(\mathbb{A} \mathbb{G}(R))$ is finite if and only if $\operatorname{Min}(R)$ is finite.
\end{abstract}

\section{Preliminary}

Researchers try to define a graph illustration for some kind of mathematical aspects. For example see [3] in the lattice literature, [13] in the measure literature and [16] in the real-valued continuous functions literature. The study of translating graph properties to algebraic properties is an interesting subject for mathematicians. The concept of zero-divisor graph of a commutative was initiated in [17]. In this article the authors let all elements of the commutative ring be vertices of the graph. The authors in [12] studied the zero-divisor graph whose vertices are the non-zero zero-divisor elements. Studying of this graph has been continued in several articles; see $[24,11,4,5,28,29]$. Also, first the

Key Words: Zero-divisor graph, Annihilating-ideal graph, Fixed-place ideal, Ring of real-valued continuous, Zariski-topology.

2010 Mathematics Subject Classification: 13A99,13A05,54C40

Received: 25.08.2020

Accepted: 30.12.2020 
annihilating-ideal graph is introduced and studied in [18] and then it has been studied in several articles; see $[19,10,2,1,26,21,27]$. In those researches some graph properties of the graphs of $\mathbb{A} \mathbb{G}(R)$ and $\Gamma(R)$ is characterized by some ring properties of $R$. In this article, we study more graph properties, for example the radius, triangulation and domination and total domination number of these graphs by some ring properties and topological properties of Zariski topology. We find the impossible assumption " $\Gamma(R)$ is a triangulated graph" in [29, Corollary 3.3] and some mistakes in the proof of $[27$, Theorem $2.2]$. In $[27$, Theorem 2.2], it is shown that the total domination of $\mathbb{A} \mathbb{G}(R)$ is equal to the cardinal of the family all maximal elements of $\mathbb{A}(R)^{*}$. By giving a counterexample, we show that [27, Theorem 2.2] is not true. Finally, by giving some facts, we correct these mistakes.

In the next section, first we recall some pertinent definitions and then give a retract of the annihilating ideal graph. Sec. 3 is devoted to the translating graph properties of these graphs to Zariski topology. Also, we note an impossible assumption in [29, Corollary 3.3]. In Sec. 4, we characterize the radius of $\Gamma(X)$ and $\mathbb{A} \mathbb{G}(X)$. In the last section, the domination number of the annihilating-ideal graph is studied and we note some mistakes in $[27$, Theorem $2.2]$.

\section{Introduction}

Throughout this article, $R$ is a commutative unitary reduced ring. $\operatorname{By} \operatorname{Spec}(R)$, we mean the set of all prime ideals of $R$. A semi-prime ideal means an ideal which is the intersection of a family of prime ideals. $R$ is called a reduced ring if the zero ideal of $R$ is semi-prime. For each ideal $I$ of $R$ and each subset $S$ of $R$, we denote the ideal $\{x \in R: S x \subseteq I\}$ by $(I: S)$. When $I=\{0\}$, we write $\operatorname{Ann}(S)$ instead of $(\{0\}: S)$ and call it the annihilator of $S$. Also, we write $\operatorname{Ann}(a)$ instead of $\operatorname{Ann}(\{a\})$. A prime ideal $P$ is said to be a minimal prime $i d e a l$ over an ideal $I$ if there are not any prime ideal strictly contained in $P$ that contains $I$. By $\operatorname{Min}(I)$, we mean the set of all minimal prime ideals over I. We use $\operatorname{Min}(R)$ instead of $\operatorname{Min}(\{0\})$. A prime ideal $P$ is called a Bourbaki associated prime divisor of an ideal $I$ if $(I: x)=P$, for some $x \in R$. We denote the set of all Bourbaki associated prime divisors of an ideal $I$ by $\mathcal{B}(I)$. It is easy to see that $\mathcal{B}(I) \subseteq \operatorname{Min}(I)$, for any ideal $I$ of a ring $R$. We use $\mathcal{B}(R)$ instead of $\mathcal{B}(\{0\})$. Let $I$ be a semi-prime ideal, then $P_{\circ} \in \operatorname{Min}(I)$ is called irredundant with respect to $I$ if $I \neq \bigcap_{P_{\circ} \neq P \in \operatorname{Min}(I)} P$. If $I$ is equal to the intersection of all irredundant ideals with respect to $I$, then we call it a fixed-place ideal. In this case, exactly by [6, Theorem 2.1], we have $I=\bigcap \mathcal{B}(I)$. If $\mathcal{B}(I)=\emptyset$, then $I$ is called an anti-fixed place ideal. We use $\mathcal{B}(R)$ instead of $\mathcal{B}(\{0\})$. For more information about the fixed-place ideals and anti fixed-place 
ideals, see $[6,7]$.

Let $G=\langle V(G), E(G)\rangle$ be an undirected graph. A vertex is called a pendant vertex if it is adjacent to just one vertex. For each pair of vertices $u$ and $v$ in $V(G)$, the length of the shortest path between $u$ and $v$, denoted by $d(u, v)$, is called the distance between $u$ and $v$. The eccentricity of a vertex $u$ of $G$ is denoted by $\operatorname{ecc}(u)$ and is defined to be maximum of $\{d(u, v): v \in$ $G\}$. The minimum of $\{\operatorname{ecc}(u): u \in G\}$, denoted by $\operatorname{Rad}(G)$, is called the radius of $G$. We say $G$ is triangulated if each vertex of $G$ is a vertex of some triangle. Two vertices $u$ and $v$ are called orthogonal if $u$ and $v$ are adjacent and there are not any vertex which is adjacent to the both vertices $u$ and $v$. A graph homomorphism $\varphi$ from a graph $G=\langle V(G), E(G)\rangle$ to a graph $H=\langle V(H), E(H)\rangle$ is a map from $V(G)$ to $V(H)$ that $\{u, v\} \in E(G)$ implies $\{f(u), f(v)\} \in E(H)$, for all pairs of vertices $u, v \in V(G)$. A retraction is a homomorphism $\varphi$ from a graph $G$ to a subgraph $H$ of $G$ such that $\varphi(v)=v$, for each vertex $v \in V(H)$. In this case, the subgraph $H$ is called a retract of $G$. A subset $D$ of vertices of a graph is called a dominating set if every vertex of the graph is either in $D$ or adjacent to some vertex of $D$. Also, a total dominating set of a graph is a family $S$ of vertices of the graph such that every vertex is adjacent to some vertex of $S$. The domination number and total domination number of a graph is the minimum cardinality of dominating and total dominating sets of the graph, respectively. We denote the domination and total domination numbers of a graph $G$ by $\operatorname{dt}(G)$ and $\mathrm{dt}_{t}(G)$, respectively. For every $u, v \in V(G)$, we denote the length of the shortest cycle containing $u$ and $v$ by $g i(u, v)$.

Suppose that $I$ and $a$ are an ideal and element of $R$, respectively. If $\operatorname{Ann}(I) \neq\{0\}$, then $I$ is called annihilating-ideal and if $\operatorname{Ann}(a) \neq\{0\}$, then $a$ is called a zero-divisor element. Let $\mathbb{A}(R)^{*}$ be the family of all non-zero annihilating-ideals and $Z(R)^{*}$ be the family of all non-zero zero-divisor elements of $R . \mathbb{A} G(R)$ is a graph with the vertices $\mathbb{A}(R)^{*}$ and two distinct vertices $I$ and $J$ are adjacent if $I J=\{0\}$. Also, $\Gamma(R)$ is a graph with vertices $Z(R)^{*}$ and two distinct vertices $a$ and $b$ are adjacent if $a b=0 . \mathbb{A} \mathbb{G}(R)$ and $\Gamma(R)$ are called the annihilating-ideal graph and the zero-divisor graph of $R$, respectively.

Throughout this article, all $Y \subseteq \operatorname{Spec}(R)$ is considered by Zariski topology; i.e., by assuming as a base for the closed sets of $Y$, the sets $h_{Y}(a)=\{P \in Y$ : $a \in P\}$. Hence, the closed sets of $Y$ are of the form $h_{Y}(I)=\bigcap_{a \in I} h_{Y}(a)=$ $\{P \in Y: I \subseteq P\}$, for some ideal $I$ in $R$. Also, we set $h_{Y}^{c}(I)=Y \backslash h_{Y}(I)$. When $Y=\operatorname{Min}(R)$, we write $h_{m}$ instead of $h_{Y}$. A point $P \in \operatorname{Spec}(R)$ is called a quasi-isolated point, if $P$ is an isolated point of $\operatorname{Min}(R)$. By [23, Theorem 2.3 and Corollary 2.4], the $\operatorname{space} \operatorname{Min}(R)$ is a Hausdorff space in which $\left\{h_{m}^{c}(a): a \in R\right\}$ is a base of clopen sets. 
In this research, $C(X)$ denotes the ring of all real-valued continuous functions on a Tychonoff space $X$ and we abbreviate $\mathbb{A}(C(X))^{*}$ and $\mathbb{A} \mathbb{G}(C(X))$ by $\mathbb{A}(X)^{*}$ and $\mathbb{A} \mathbb{G}(X)$, respectively. We denote the set of all isolated points of $X$, by $I(X)$. A space $X$ is called almost discrete, if $\overline{I(X)}=X$.

The reader is referred to $[14,30,31,22,20]$ for undefined terms and notations.

For each subset $S$ of $R$, let $P_{S}$ be the intersection of all minimal prime ideals containing $S$. An ideal $I$ in $R$ is said to be strongly $z^{\circ}$-ideal (or briefly $s z^{\circ}$-ideal) if $P_{F} \subseteq I$, for every finite subset $F$ of $I$. Since the intersection of a family of strong $z^{\circ}$-ideals is a strong $z^{\circ}$-ideal, the smallest strong $z^{\circ}$-ideal containing an ideal $I$ exists, and we denote it by $I_{s z^{\circ}}$. For more details about the strong $z^{\circ}$-ideals, see $[25,9,8]$.

Lemma 2.1. Let $I$ and $J$ be two vertices of $\mathbb{A} \mathbb{G}(R)$. $I$ is adjacent to $J$ if and only if $I_{s z^{\circ}}$ is adjacent to $J_{s z^{\circ}}$.

Proof. $\Rightarrow)$. Suppose that $a \in I_{s z^{\circ}}$ and $b \in J_{s z^{\circ}}$, then by [8, Proposition 7.5], finite subsets $F$ of $I$ and $G$ of $J$ exist such that $h_{m}(G) \subseteq h_{m}(a)$ and $h_{m}(H) \subseteq$ $h_{m}(b)$. Since $I$ is adjacent to $J$, it follows that $I J=\{0\}$, so $G H=\{0\}$, this implies that $\operatorname{Min}(R)=h_{m}(G H)=h_{m}(G) \cup h_{m}(H) \subseteq h_{m}(a) \cup h_{m}(b)=h_{m}(a b)$, thus $h_{m}(a b)=\operatorname{Min}(R)$, hence $a b \in k h_{m}(a b)=\{0\}$, and, therefore $a b=0$. This shows that $I_{s z^{\circ}} J_{s z^{\circ}}=\{0\}$ and thus $I_{s z^{\circ}}$ is adjacent to $J_{s z^{\circ}}$.

$\Leftarrow)$. It is clear.

Proposition 2.2. The family of all sz $z^{\circ}$-ideals of $\mathbb{A}(R)^{*}$ is a retract of $\mathbb{A} \mathbb{G}(R)$.

Proof. Suppose that $I \in \mathbb{A}(R)^{*}$, so $J \in \mathbb{A}(R)^{*}$ exists such that $I J=\{0\}$. By Lemma 2.1, we have $I_{s z^{\circ}}$ is adjacent to $J_{s z^{\circ}}$. Since $0 \neq I \subseteq I_{s z^{\circ}} \subseteq$ $\operatorname{Ann}\left(J_{s z^{\circ}}\right) \subseteq \operatorname{Ann}(J) \neq R$, it follows that $I_{s z^{\circ}} \in \mathbb{A}(R)^{*}$. This shows that $\varphi$ from $\mathbb{A}(R)^{*}$ to the family of all $s z^{\circ}$-ideals of $\mathbb{A}(R)^{*}$, defined by $\varphi(I)=I_{s z^{\circ}}$, is a retraction, and, therefore the family of all $s z^{\circ}$-ideals of $\mathbb{A}(R)^{*}$ is a retract of $\mathbb{A} \mathbb{G}(R)$.

\section{Zariski Topology}

In this section, we give Zariski topological characterization of elements of $\Gamma(R)$ and $\mathbb{A} \mathbb{G}(R)$, then we characterize the adjacency, distance, orthogonality, eccentricity and triangulation. Also, it is shown that the radius of these graphs are strictly greater that 1.

Lemma 3.1. Let $Y \subseteq \operatorname{Spec}(R)$ and $\bigcap Y=\{0\}$. Then

(a) $a=0$ if and only if $h_{Y}(a)=Y$, for each $a \in R$. 
(b) $\operatorname{Ann}(a) \neq 0$ if and only if $\overline{h_{Y}^{c}(a)} \neq Y$, for each $a \in R$.

(c) $I=\{0\}$ if and only if $h_{Y}(I)=Y$, for each ideal $I$ of $R$.

(d) $I$ is an annihilating-ideal if and only if $\overline{h_{Y}^{c}(I)} \neq Y$.

(e) For each $a, b \in Z(R)^{*}$, a is adjacent to $b$ if and only if $h_{Y}^{c}(a) \cap h_{Y}^{c}(b)=\emptyset$.

(f) For each $I, J \in \mathbb{A}(R)^{*}, I$ is adjacent to $J$ if and only if $h_{Y}^{c}(I) \cap h_{Y}^{c}(J)=$ $\emptyset$.

Proof. (a) and (c). Since $\bigcap Y=\{0\}$, they are clear.

(b). Since $\operatorname{Ann}(a)=k h_{Y}^{c}(a)$, it follows that $\operatorname{Ann}(a) \neq\{0\}$ if and only if $k h_{Y}^{c}(a) \neq\{0\}$, and it is equivalent to say that $h k h_{Y}^{c}(a) \neq Y$, because $\bigcap Y=\{0\}$, and, therefore they are equivalent to $\overline{h_{Y}^{c}(I)} \neq Y$.

(d). The proof is analogously similar to the proof of part (b).

(e) and (f). They are evident.

In [29, Proposition 2.2], the concept of distance in $\Gamma(R)$ is characterized by Zariski topology on $\operatorname{Spec}(R)$. In the following proposition, we generalize this characterization by every reduced family of prime ideals and also we characterize the concept of distance in $\mathbb{A} \mathbb{G}$.

Proposition 3.2. Let $I, J \in \mathbb{A}(R)^{*}, a, b \in Z(R)^{*}, Y \subseteq \operatorname{Spec}(R)$ and $\bigcap Y=$ $\{0\}$. Then

(a) $d(a, b)=1$ if and only if $h_{Y}^{c}(a) \cap h_{Y}^{c}(b)=\emptyset$.

(b) $d(a, b)=2$ if and only if $h_{Y}^{c}(a) \cap h_{Y}(b) \neq \emptyset$ and $h_{Y}^{c}(a) \cup h_{Y}^{c}(b)$ is not dense in $Y$.

(c) $d(a, b)=3$ if and only if $h_{Y}^{c}(a) \cap h_{Y}^{c}(b) \neq \emptyset$ and $h_{Y}^{c}(a) \cup h_{Y}^{c}(b)$ is dense in $Y$.

(d) $d(I, J)=1$ if and only if $h_{Y}^{c}(I) \cap h_{Y}^{c}(J)=\emptyset$.

(e) $d(I, J)=2$ if and only if $h_{Y}^{c}(I) \cap h_{Y}^{c}(J) \neq \emptyset$ and $h_{Y}^{c}(I) \cup h_{Y}^{c}(J)$ is not dense in $Y$.

(f) $d(I, J)=3$ if and only if $h_{Y}^{c}(I) \cap h_{Y}^{c}(J) \neq \emptyset$ and $h_{Y}^{c}(I) \cup h_{Y}^{c}(J)$ is dense in $Y$. 
Proof. (a) and (d). They are clear, by Lemma 3.1.

$(\mathrm{b} \Rightarrow)$. By Lemma 3.1, we have $h_{Y}^{c}(a) \cap h_{Y}^{c}(b) \neq \emptyset$. By the assumption, there is an element $c \in Z(R)^{*}$ such that $c$ is adjacent to the both vertices $a$ and $b$. Now Lemma 3.1 implies that

$$
h_{Y}^{c}(a) \cap h_{Y}^{c}(c)=h_{Y}^{c}(a) \cap h_{Y}^{c}(c)=\emptyset \quad \Rightarrow \quad h_{Y}^{c}(a) \cup h_{Y}^{c}(b) \subseteq h_{Y}(c)
$$

Since $c \neq 0$, by Lemma 3.1, it follows that $h_{Y}(c) \neq Y$. Since $h_{Y}(c)$ is closed, by $(*)$, it follows that $h_{Y}^{c}(a) \cup h_{Y}^{c}(b)$ is not dense in $Y$.

$(\mathrm{b} \Leftarrow)$. Part (a) concludes that $d(a, b)>1$. Since $\left\{h_{Y}^{c}(c): c \in R\right\}$ is a base for Zariski topology, by the assumption, it follows that there is some $c \in R$ such that $h_{Y}^{c}(a) \cup h_{Y}^{c}(b) \subseteq h_{Y}(c) \subset Y$, so $h_{Y}^{c}(a) \cap h_{Y}^{c}(c)=h_{Y}^{c}(a) \cap h_{Y}^{c}(c)=\emptyset$, $Y \neq h_{Y}(a)$ and $\overline{h_{Y}^{c}(c)} \neq Y$, hence $c \in Z(R)^{*}$ and $c$ is adjacent to the both vertices $a$ and $b$ and thus $d(a, b)=2$.

(c). It deduces from parts (a), (b) and [12, Theorem 2.2].

(e). By the fact that $\left\{h_{Y}^{c}(K): K\right.$ is an ideal of $\left.R\right\}$ is a base for Zariski topology, it is similar to part (b)

(f). It concludes from parts (d), (e) and [18, Theorem 7.1].

Theorem 3.3. Let $I, J \in \mathbb{A}(R)^{*}, a, b \in Z(R)^{*}, Y \subseteq \operatorname{Spec}(R)$ and $\bigcap Y=\{0\}$. Then

(a) Two vertices $I$ and $J$ are orthogonal if and only if $h_{Y}^{c}(I) \cap h_{Y}^{c}(J)=\emptyset$ and $\overline{h_{Y}^{c}(I) \cup h_{Y}^{c}(J)}=Y$.

(b) Two vertices $a$ and $b$ are orthogonal if and only if $h_{Y}^{c}(a) \cap h_{Y}^{c}(b)=\emptyset$ and $\overline{h_{Y}^{c}(a) \cup h_{Y}^{c}(b)}=Y$.

Proof. ( $\mathrm{a} \Rightarrow$ ). By the assumption and Lemma 3.1, we have $I$ is adjacent to $J$ and thus $h_{Y}^{c}(I) \cap h_{Y}^{c}(J)=\emptyset$. If $\overline{h_{Y}^{c}(I) \cup h_{Y}^{c}(J)} \neq Y$, since $\left\{h_{Y}^{c}(K)\right.$ : $K$ is an ideal of $R\}$ is a base for Zariski topology, it follows that there is some ideal $K$ of $R$ such that $h_{Y}^{c}(K) \cap\left[h_{Y}^{c}(I) \cup h_{Y}^{c}(J)\right]=\emptyset$, so $h_{Y}^{c}(K) \cap h_{Y}^{c}(I)=$ $h_{Y}^{c}(K) \cap h_{Y}^{c}(J)=\emptyset, h_{Y}^{c}(K) \neq Y$ and $\overline{h_{Y}^{c}(K)} \neq Y$, hence $K \in \mathbb{A}(R)^{*}$, by Lemma 3.1, and $K$ is adjacent to the both vertices $I$ and $J$, by Lemma 3.1, which contradicts the assumption and thus $\overline{h_{Y}^{c}(I) \cup h_{Y}^{c}(J)}=Y$.

$(\mathrm{a} \Leftarrow)$. By the assumption and Lemma 3.1 , we have $h_{Y}^{c}(I) \cap h_{Y}^{c}(J)=\emptyset$. On the contrary, suppose that there is an $K \in \mathbb{A}(R)^{*}$ such that $K$ is adjacent to the both vertices $I$ and $J$, then $h_{Y}^{c}(K) \cap\left[h_{Y}^{c}(I) \cup h_{Y}^{c}(J)\right]=\left[h_{Y}^{c}(K) \cap h_{Y}^{c}(I)\right] \cup$ $\left[h_{Y}^{c}(K) \cap h_{Y}^{c}(J)\right]=\emptyset$, by Lemma 3.1. Since $K \in \mathbb{A}(R)^{*}$, by Lemma 3.1, it follows that $h_{Y}^{c}(K) \neq \emptyset$, and, therefore $\overline{h_{Y}^{c}(I) \cup h_{Y}^{c}(J)} \neq Y$, which contradicts the assumption.

(b). By the fact that $\left\{h_{Y}^{c}(c): c \in R\right\}$ is a base for Zariski topology, the proof is similar to part (a). 
Suppose that $\bigcap Y=\{0\}$. Since for every $I \in \mathbb{A}(R)^{*}$, it follows that $I$ and $\operatorname{Ann}(I)$ are orthogonal and thus, we have $\overline{h_{Y}^{c}(I) \cap h_{Y}^{c}(\operatorname{Ann}(I))}=Y$, by the above theorem. Similarly, for every $a \in Z(R)^{*}$ and $b \in \operatorname{Ann}(a)$, we have $\overline{h_{Y}^{c}(a) \cup h_{Y}^{c}(b)}=Y$.

Theorem 3.4. Suppose that $I \in \mathbb{A}(R)^{*}, a \in Z(R)^{*}, Y \subseteq \operatorname{Min}(R)$ and $\bigcap Y=$ $\{0\}$. Then

(a) $\operatorname{ecc}(I)>1$, for every $I \in \mathbb{A}(R)^{*}$.

(b) $\operatorname{ecc}(I)=2$ if and only if $h_{Y}^{c}(I)$ is singleton.

(c) $\operatorname{ecc}(I)=3$ if and only if $h_{Y}^{c}(I)$ is not singleton.

(d) $\operatorname{ecc}(a)>1$, for every $a \in Z(R)^{*}$.

(e) $\operatorname{ecc}(a)=2$ if and only if $h_{Y}^{c}(a)$ is singleton.

$(f) \operatorname{ecc}(a)=3$ if and only if $h_{Y}^{c}(a)$ is not singleton.

Proof. Since $R$ is not an integral domain and $\bigcap Y=\{0\}$, it follows that $|Y| \geqslant 2$.

$(\mathrm{c} \Rightarrow)$. By the assumption there is some $J \in \mathbb{A}(R)^{*}$ such that $d(I, J)=3$. Lemma 3.2 implies that $h_{Y}^{c}(I) \cap h_{Y}^{c}(J)=\emptyset$ and $\overline{h_{Y}^{c}(I) \cup h_{Y}^{c}(J)}=Y$. On the contrary, suppose that $h_{Y}^{c}(I)$ is singleton, then $h_{Y}^{c}(I) \subseteq h_{Y}^{c}(J)$, and, therefore $\overline{h_{Y}^{c}(J)}=\overline{h_{Y}^{c}(I) \cup h_{Y}^{c}(J)}=Y$. Hence $J \notin \mathbb{A}(R)^{*}$, by Lemma 3.1, which is a contradiction.

$(\mathrm{c} \Leftarrow)$. By the assumption, there are distinct prime ideals $P$ and $Q$ in $h_{Y}^{c}(I)$. Since $Y \subseteq \operatorname{Min}(R)$ is Hausdorff and $\left\{h_{Y}^{c}(K): K\right.$ is an ideal of $\left.R\right\}$ is a base for $Y$, there are ideals $J$ and $K$ such that $h_{Y}^{c}(J), h_{Y}^{c}(K) \subseteq h_{Y}^{c}(I)$, $P \in h_{Y}^{c}(J), Q \in h_{Y}^{c}(K)$ and $h_{Y}^{c}(J) \cap h_{Y}^{c}(K)=\emptyset$. Thus

$$
\begin{aligned}
h_{Y}^{c}(J+\operatorname{Ann}(I)) \cap h_{Y}^{c}(K) & =\left[h_{Y}^{c}(J) \cup h_{Y}^{c}(\operatorname{Ann}(I))\right] \cap h_{Y}^{c}(K) \\
& \subseteq\left[h_{Y}^{c}(J) \cap h_{Y}^{c}(K)\right] \cup\left[h_{Y}^{c}(\operatorname{Ann}(I)) \cap h_{Y}^{c}(I)\right]=\emptyset .
\end{aligned}
$$

Hence $h_{Y}^{c}(J+\operatorname{Ann}(I)) \neq Y$ and $\overline{h_{Y}^{c}(J+\operatorname{Ann}(I))} \neq Y$, and, therefore we have $J+\operatorname{Ann}(I) \in \mathbb{A}(R)^{*}$. Since

$$
h_{Y}^{c}(I) \cap h_{Y}^{c}(J+\operatorname{Ann}(I)) \supseteq h_{Y}^{c}(I) \cap h_{Y}^{c}(J)=h_{Y}^{c}(I) \neq \emptyset
$$

and

$$
\overline{h_{Y}^{c}(I) \cap h_{Y}^{c}(J+\operatorname{Ann}(I))} \supseteq \overline{h_{Y}^{c}(I) \cap h_{Y}^{c}(\operatorname{Ann}(I))}=Y,
$$

by Proposition 3.2 , we have $d(I, J+\operatorname{Ann}(I))=3$, and, therefore $\operatorname{ecc}(I)=3$, by $[18$, Theorem 7.1$]$. 
(a). Suppose that there is some $I \in \mathbb{A}(R)^{*} \operatorname{such}$ that $\operatorname{ecc}(I)=1$. Part (c) concludes that $h_{Y}^{c}(I)$ is singleton, so there is some $P \in Y$ such that $h_{Y}^{c}(I)=$ $\{P\}$, hence $\operatorname{Ann}(I)=P$ and thus $\{0\} \neq I \subseteq \operatorname{Ann}(P)$. Since $\operatorname{ecc}(I)=1$, it follows that $I$ is adjacent to $\operatorname{Ann}(P)$, consequently $I \operatorname{Ann}(P)=\{0\}$, this implies that for every $a \in I$, we have $a^{2} \in I \operatorname{Ann}(P)=\{0\}$, and, therefore $a^{2}=0$. Since $R$ is reduced, it follows that $a=0$ and consequently $I=\{0\}$, which is a contradiction.

(b). By parts (a), (c) and [18, Theorem 7.1], it is clear.

The proof of parts (d), (e) and (f) are similar to parts (a), (b) and (c), respectively.

The following corollary is an immediate consequence of the above theorem.

Corollary 3.5. $\operatorname{Rad} \Gamma(R)>1$ and $\operatorname{Rad} \mathbb{A} G(R)>1$.

Lemma 3.6. Let $a \in Z(R)^{*}, I \in \mathbb{A}(R)^{*}, Y \subseteq \operatorname{Min}(R)$ and $\bigcap Y=\{0\}$. Then

(a) a is a vertex of a triangle if and only if $h_{Y}(a)$ is not singleton.

(b) I is a vertex of a triangle if and only if $h_{Y}(I)^{\circ}$ is not singleton.

Proof. $(\mathrm{a} \Rightarrow)$. By the assumption, there are vertices $b, c \in \mathbb{A}(R)^{*}$ such that $a, b$ and $c$ are pairwise distinct adjacent vertices. Thus $h_{Y}^{c}(a), h_{Y}^{c}(b)$ and $h_{Y}^{c}(c)$ are pairwise disjoint nonempty sets, by Lemma 3.1, hence $h_{Y}^{c}(b) \cup h_{Y}^{c}(c) \subseteq h_{Y}(a)$ and $\left|h_{Y}^{c}(b) \cup h_{Y}^{c}(c)\right| \geqslant 2$. Since $h_{Y}^{c}(b) \cup h_{Y}^{c}(c)$ is open, it follows that $h_{Y}(a)$ is not singleton.

$(\mathrm{a} \Leftarrow)$. Suppose that $P$ and $Q$ are distinct elements of $h_{Y}(a)$. Since $Y \subseteq \operatorname{Min}(R)$ is Hausdorff, $h_{Y}(a)$ is open and $\left\{h_{Y}^{c}(x): x \in R\right\}$ is a base for $Y$, it follows that there are $b, c \in R$ such that $P \in h_{Y}^{c}(b) \subseteq h_{Y}(a), Q \in$ $h_{Y}^{c}(c) \subseteq h_{Y}(a)$ and $h_{Y}^{c}(b) \cap h_{Y}^{c}(c)=\emptyset$, and, therefore $h_{Y}^{c}(a), h_{Y}^{c}(b)$ and $h_{Y}^{c}(c)$ are pairwise disjoint nonempty sets which are not dense in $Y$. Now Lemma 3.1 implies that $b, c \in \mathbb{A}(R)^{*}$ and Theorem 3.1 concludes that $a, b$ and $c$ are pairwise distinct adjacent vertices and thus $a$ is a vertex of a triangle.

(b). It is similar to part (a).

Proposition 3.7. Suppose that $a, b \in Z(R)^{*}$ are not pendant vertices, $Y \subseteq$ $\operatorname{Min}(R)$ and $\bigcap Y=\{0\}$. Then

(a) $h_{Y}^{c}(a) \cap h_{Y}^{c}(b)=\emptyset$ and $\overline{h_{Y}^{c}(a) \cup h_{Y}^{c}(b)} \neq Y$ if and only if $\operatorname{gi}(a, b)=3$.

(b) If $2 \notin Z(R), h_{Y}^{c}(a) \cap h_{Y}^{c}(b)=\emptyset$ and $\overline{h_{Y}^{c}(a) \cup h_{Y}^{c}(b)}=Y$, then $\operatorname{gi}(a, b)=$ 4 .

(c) Suppose that $h_{Y}^{c}(a) \cap h_{Y}^{c}(b) \neq \emptyset$. Then $\overline{h_{Y}^{c}(a) \cup h_{Y}^{c}(b)} \neq Y$ if and only if $\operatorname{gi}(a, b)=4$. 
(d) Suppose that $2 \notin Z(R)$ and $h_{Y}^{c}(a) \cap h_{Y}^{c}(b) \neq \emptyset$. Then $\overline{h_{Y}^{c}(a) \cup h_{Y}^{c}(b)}=Y$ if and only if $\operatorname{gi}(a, b)=6$.

Proof. By Lemma 3.1, the proof is similar to [29, Theorem 3.4].

Theorem 3.8. Suppose that $I, J \in \mathbb{A}(R)^{*}$ and they are not pendant vertices. The following statements hold.

(a) $h_{Y}^{c}(I) \cap h_{Y}^{c}(J)=\emptyset$ and $\overline{h_{Y}^{c}(I) \cup h_{Y}^{c}(J)} \neq Y$ if and only if $\operatorname{gi}(I, J)=3$.

(b) If $h_{Y}^{c}(I) \cap h_{Y}^{c}(J)=\emptyset$ and $\overline{h_{Y}^{c}(I) \cup h_{Y}^{c}(J)}=Y$, then $\operatorname{gi}(I, J)=4$.

(c) If $h_{Y}^{c}(I) \cap h_{Y}^{c}(J) \neq \emptyset$ and $\overline{h_{Y}^{c}(I)}=\overline{h_{Y}^{c}(J)}$, then $\operatorname{gi}(I, J)=4$.

(d) If $h_{Y}^{c}(I) \cap h_{Y}^{c}(J) \neq \emptyset$ and $\overline{h_{Y}^{c}(I)} \neq \overline{h_{Y}^{c}(J)}$ and $Y \backslash \overline{h_{Y}^{c}(I) \cup h_{Y}^{c}(J)}$ is not singleton, then $\operatorname{gi}(I, J)=4$.

(e) If $h_{Y}^{c}(I) \cap h_{Y}^{c}(J) \neq \emptyset, \overline{h_{Y}^{c}(I)} \neq \overline{h_{Y}^{c}(J)}$ and $Y \backslash \overline{h_{Y}^{c}(I) \cup h_{Y}^{c}(J)}$ is singleton, then $4 \leqslant \operatorname{gi}(I, J) \leqslant 5$.

(f) If $\operatorname{gi}(I, J)=5$, then $h_{Y}^{c}(I) \cap h_{Y}^{c}(J) \neq \emptyset, \overline{h_{Y}^{c}(I)} \neq \overline{h_{Y}^{c}(J)}$ and $Y \backslash$ $\overline{h_{Y}^{c}(I) \cup h_{Y}^{c}(J)}$ is singleton.

Proof. ( $\Rightarrow$ ). By Lemma 3.1, $I$ is adjacent to $J$ and by Theorem 3.3, $I$ and $J$ are not orthogonal. Thus gi $(I, J)=3$.

(a $\Leftarrow$ ). By the assumption, $I$ is adjacent to $J$ and, $I$ and $J$ are not orthogonal, so by Lemma 3.1, we have $h_{Y}^{c}(I) \cap h_{Y}^{c}(J)=\emptyset$ and, by Proposition 3.3 , we have $\overline{h_{Y}^{c}(I) \cup h_{Y}^{c}(J)} \neq Y$.

(b). By the assumption, we have $I J=\{0\}$ and we can see easily that $h_{Y}(I)^{\circ} \cap h_{Y}(J)^{\circ}=\emptyset$. We know that $h_{Y}^{c}(\operatorname{Ann}(I)) \subseteq h_{Y}(I)^{\circ}$ and $h_{Y}^{c}(\operatorname{Ann}(J)) \subseteq$ $h_{Y}(J)^{\circ}$, so $h_{Y}^{c}(\operatorname{Ann}(I)) \cap h_{Y}^{c}(\operatorname{Ann}(J))=\emptyset$. Now Lemma 3.1 concludes that $\operatorname{Ann}(I) \operatorname{Ann}(J)=\{0\}$. Since $I$ and $J$ are not pendant vertices, there are $I_{1}, J_{1} \in \mathbb{A}(X)^{*}$ such that $I$ is adjacent to $I_{1} \neq J$ and $J$ is adjacent to $J_{1} \neq I$, so $I I_{1}=J J_{1}=\{0\}$, thus $I_{1} \subseteq \operatorname{Ann}(I)$ and $J_{1} \subseteq \operatorname{Ann}(J)$, hence $I_{1} J_{1} \subseteq$ $\operatorname{Ann}(I) \operatorname{Ann}(J)=\{0\}$, and, therefore $I_{1} J_{1}=\{0\}$. Consequently, $I-J-J_{1}-$ $I_{1}-I$ is a cycle. The assumption and part (a) conclude that gi $(I, J)>3$ and thus $\operatorname{gi}(I, J)=4$.

(c). We conclude from the assumption and part (a) that gi $(I, J) \geqslant 4$. Clearly $\operatorname{Ann}(I), \operatorname{Ann}(J) \in \mathbb{A}(R)^{*}$. Since $\overline{h_{Y}^{c}(I)}=\overline{h_{Y}^{c}(J)}$, it follows that $h_{Y}^{c}(I) \cap h_{Y}^{c}(\operatorname{Ann}(J)) \subseteq \overline{h_{Y}^{c}(I)} \cap h_{Y}(J)^{\circ}=\overline{h_{Y}^{c}(I)} \cap{\overline{h_{Y}^{c}(J)}}^{c}=\overline{h_{Y}^{c}(I)} \cap{\overline{h_{Y}^{c}(I)}}^{c}=\emptyset$, so, by Lemma 3.1, we have $I \operatorname{Ann}(J)=\{0\}$. Similarly, we can show that $J \operatorname{Ann}(I)=\{0\}$. If $\operatorname{Ann}(I) \neq \operatorname{Ann}(J)$, then $I-\operatorname{Ann}(I)-J-\operatorname{Ann}(J)-I$ is a cycle, and, therefore gi $(I, J)=4$. Now we suppose that $\operatorname{Ann}(I)=\operatorname{Ann}(J)$. Since $I$ is adjacent to $\operatorname{Ann}(I)$ and $I$ is not a pendant vertex, it follows there 
is some vertex $I_{1} \in \mathbb{A}(X)^{*}$ distinct from $\operatorname{Ann}(I)$ such that $I$ is adjacent to $I_{1}$, then $I_{1} I=\{0\}$, so $I_{1} \subseteq \operatorname{Ann}(I)=\operatorname{Ann}(J)$, and, therefore $I_{1} J=\{0\}$. Consequently, $I-\operatorname{Ann}(I)=\operatorname{Ann}(J)-J-I_{1}-I$ is a cycle and thus $\operatorname{gi}(I, J)=4$.

(d). We can conclude from the assumption and part (a) that gi $(I, J) \geqslant 4$. Since $\left\{h_{Y}^{c}(K): K\right.$ is an ideal of $\left.R\right\}$ is a base for $Y, Y$ is Hausdorff and $Y$ V $\overline{h_{Y}^{c}(I) \cap h_{Y}^{c}(J)}$ is not singleton, it follows that there are two distinct ideals $K_{1}$ and $K_{2}$ such that $h_{Y}^{c}\left(K_{1}\right) \cap\left(h_{Y}^{c}(I) \cup h_{Y}^{c}(J)\right)=h_{Y}^{c}\left(K_{2}\right) \cap\left(h_{Y}^{c}(I) \cup h_{Y}^{c}(J)\right)=\emptyset$. Hence $h_{Y}^{c}(I) \cap h_{Y}^{c}\left(K_{1}\right)=h_{Y}^{c}\left(K_{1}\right) \cap h_{Y}^{c}(J)=h_{Y}^{c}(J) \cap h_{Y}^{c}\left(K_{2}\right)=h_{Y}^{c}\left(K_{2}\right) \cap$ $h_{Y}^{c}(I)=\emptyset$. Then, by Lemma 3.1 , we have $K_{1}, K_{2} \in \mathbb{A}(R)^{*}$ and $I-K_{1}-J-$ $K_{2}-I$ is a cycle. Consequently, $\operatorname{gi}(I, J)=4$.

(e). By part (a), we have gi $(I, J) \geqslant 4$. Since $Y \backslash \overline{h_{Y}^{c}(I) \cup h_{Y}^{c}(J)} \neq Y$ and $\left\{h_{Y}^{c}(K): K\right.$ is an ideal of $\left.R\right\}$ is a base for $Y$, it follows that there is some ideal $K_{1}$ of $R$ such that $h_{Y}^{c}\left(K_{1}\right) \cap\left[h_{Y}^{c}(I) \cup h_{Y}^{c}(J)\right]=\emptyset$, so $h_{Y}^{c}\left(K_{1}\right) \cap h_{Y}^{c}(I)=$ $h_{Y}^{c}\left(K_{1}\right) \cap h_{Y}^{c}(J)=\emptyset$. By Lemma 3.1 , we have $K_{1} \in \mathbb{A}(R)^{*}$ and $K_{1}$ is adjacent to the both vertices $I$ and $J$. If there is some $K_{2} \in \mathbb{A}(R)^{*}$ distinct from $K_{1}$ such that $h_{Y}^{c}\left(K_{1}\right)=h_{Y}^{c}\left(K_{2}\right)$, then $K_{2}$ also is adjacent to the both vertices $I$ and $J$. Thus gi $(I, J)=4$. Now suppose that $h_{Y}^{c}(K)=h_{Y}^{c}\left(K_{1}\right)$ implies that $K=K_{1}$. If $h_{Y}^{c}(I) \subseteq \overline{h_{Y}^{c}(J)}$, then $\overline{h_{Y}^{c}(I)} \subseteq \overline{h_{Y}^{c}(J)}$, so $Y \backslash \overline{h_{Y}^{c}(I) \cup h_{Y}^{c}(J)}=Y \backslash \overline{h_{Y}^{c}(J)}$, and, therefore, by the assumption, $Y \backslash \overline{h_{Y}^{c}(J)}$ is singleton. Since $J$ is not a pendant vertex, there is some vertex $K_{2}$ such that $K_{2}$ is adjacent to $J$, thus, by Lemma 3.1, we have $h_{Y}^{c}\left(K_{2}\right) \cap h_{Y}^{c}(J)=\emptyset$, so $h_{Y}^{c}\left(K_{2}\right) \cap \overline{h_{Y}^{c}(J)}=\emptyset$ and thus $h_{Y}^{c}\left(K_{2}\right) \subseteq Y \backslash \overline{h_{Y}^{c}(J)}$. By Lemma 3.1, we have $h_{Y}^{c}\left(K_{2}\right) \neq \emptyset$ and therefore $h_{Y}^{c}\left(K_{2}\right)=Y \backslash \overline{h_{Y}(J)}$. Similarly, we can show that $h_{Y}^{c}\left(K_{1}\right)=Y \backslash \overline{h_{Y}(J)}$, hence $h_{Y}^{c}\left(K_{1}\right)=h_{Y}^{c}\left(K_{2}\right)$, which is a contradiction. Hence $h_{Y}^{c}(I) \nsubseteq \overline{h_{Y}^{c}(J)}$. Similarly, one can show that $h_{Y}^{c}(J) \nsubseteq \overline{h_{Y}^{c}(I)}$ and thus $h_{Y}^{c}(I) \backslash \overline{h_{Y}^{c}(J)}$ and $h_{Y}^{c}(J) \backslash \overline{h_{Y}^{c}(I)}$ are disjoint nonempty open sets. Since $\left\{h_{Y}^{c}(K): K\right.$ is an ideal of $\left.R\right\}$ is a base for $Y$, there are distinct ideals $K_{2}$ and $K_{3}$ such that $h_{Y}^{c}\left(K_{2}\right) \subseteq h_{Y}^{c}(I) \backslash \overline{h_{Y}^{c}(J)}$ and $h_{Y}^{c}\left(K_{3}\right) \subseteq h_{Y}^{c}(J) \backslash \overline{h_{Y}^{c}(I)}$. Consequently, $h_{Y}^{c}(J) \cap h_{Y}^{c}\left(K_{2}\right)=h_{Y}^{c}\left(K_{2}\right) \cap$ $h_{Y}^{c}\left(K_{3}\right)=h_{Y}^{c}\left(K_{3}\right) \cap h_{Y}^{c}(I)=\emptyset$. By Lemma 3.1, we have $K_{2}, K_{3} \in \mathbb{A}(R)^{*}$ and $I-K_{1}-J-K_{2}-K_{3}-I$ is a cycle, and, therefore gi $(I, J) \leqslant 5$.

(f). It is clear, by parts (a)-(e).

Suppose that $R=\mathbb{Z} \times \mathbb{Z} \times \mathbb{Z} \times \mathbb{Z}, I=\{0\} \times \mathbb{Z} \times \mathbb{Z} \times\{0\}, J=\mathbb{Z} \times\{0\} \times \mathbb{Z} \times\{0\}$, $R^{\prime}=\mathbb{R} \times \mathbb{R} \times \mathbb{R} \times \mathbb{R}, I^{\prime}=\{0\} \times \mathbb{R} \times \mathbb{R} \times\{0\}$ and $J=\mathbb{R} \times\{0\} \times \mathbb{R} \times\{0\}$. Then the both pair vertices $I, J \in \mathbb{A}(R)^{*}$ and $I^{\prime}, J^{\prime} \in \mathbb{A}\left(R^{\prime}\right)^{*}$ satisfy the conditions of part (e) of the above theorem but it is seen readily that gi $(I, J)=4$ and $\operatorname{gi}\left(I^{\prime}, J^{\prime}\right)=5$.

Now we can conclude the following corollary from the above theorem and [6, Corollary 4.2].

Corollary 3.9. If there are $I, J \in \mathbb{A}(R)^{*}$ such that $\operatorname{gi}(I, J)=5$, then the 
following equivalent conditions hold

(a) $\operatorname{Min}(R)$ has an isolated point.

(b) $\mathcal{B}(R) \neq \emptyset$.

\section{Radius and Triangulation}

This section is devoted to study of the radius and the triangulation of $\Gamma(R)$ and $\mathbb{A} \mathbb{G}(R)$. We show that the concept of the anti fixed-place ideal plays the main role in this studying.

Theorem 4.1. The following statements are equivalent.

(a) $\operatorname{Rad} \Gamma(R)=3$.

(b) $\operatorname{Rad} \mathbb{A} G(R)=3$.

(c) The zero ideal of $R$ is an anti fixed-place ideal.

(d) The $\operatorname{Min}(R)$ does not have any isolated point.

Proof. $(\mathrm{a}) \Rightarrow(\mathrm{b})$. Suppose that $\operatorname{Rad} \mathbb{A} \mathbb{G}(R) \neq 3$, then, by Corollary 3.5 and [19, Theorem 1.4], there is some $I \in \mathbb{A}(R)^{*}$ such that $\operatorname{ecc}(I)=2$, hence, by Theorem 3.4, there is some $P \in \operatorname{Min}(R)$ such that $h_{m}^{c}(I)=\{P\}$ and thus $\operatorname{Ann}(I)=P$. Set $0 \neq a \in I$, then $\emptyset \neq h_{m}^{c}(a) \subseteq h_{m}^{c}(I)=\{P\}$, so $h_{m}^{c}(a)=\{P\}$, and, therefore $\operatorname{ecc}(a)=2$, by Theorem 3.4. Consequently, $\operatorname{Rad} \Gamma(R) \neq 3$.

(b) $\Rightarrow(\mathrm{c})$. Suppose that the zero ideal of $R$ is not an anti fixed-place ideal, then there is an affiliated prime ideal $P$, hence $a \in Z(R)^{*}$ exists such that $\operatorname{Ann}(a)=P$, this implies that $\langle a\rangle \in \mathbb{A}(R)^{*}$ and $h_{m}^{c}(\langle a\rangle)=h_{m}^{c}(a)=\{P\}$, and, therefore $\operatorname{Rad} \mathbb{A} G(R) \neq 3$, by Theorem 3.4 .

$(\mathrm{c}) \Rightarrow(\mathrm{a})$. Suppose that $\operatorname{Rad} \Gamma(R) \neq 3$. Then Corollary 3.5 and [18] imply that some $a \in Z(R)^{*}$ exists such that $\operatorname{ecc}(a)=2$, hence, by Theorem 3.5, there is some $P \in \operatorname{Min}(R)$ such that $h_{m}^{c}(a)=\{P\}$, thus $\operatorname{Ann}(a)=P$, hence $P$ is an affiliated prime ideal, so $P \in \mathcal{B}(R) \neq \emptyset$, and, therefore the zero ideal of $R$ is not an anti fixed-place ideal.

$(\mathrm{c}) \Leftrightarrow(\mathrm{d})$. It implies from [6, Corollary 4.3].

The following corollary is an immediate consequence of [19, Theorem 1.4], the above theorem and Corollary 3.5.

Corollary 4.2. The following statements are equivalent.

(a) $\operatorname{Rad} \Gamma(R)=2$. 
(b) $\operatorname{Rad} \mathbb{A} \mathbb{G}(R)=2$.

(c) The zero ideal of $R$ is not an anti fixed-place ideal.

(d) The $\operatorname{Min}(R)$ has an isolated point.

Now we can conclude the following corollary from the above theorem and corollary.

Corollary 4.3. $\operatorname{Rad} \Gamma(R)=\operatorname{Rad} \mathbb{A} G(R)$.

Corollary 4.4. Suppose that $X$ is a Tychonoff topological space. Then

$$
\operatorname{Rad} \Gamma(X)=\operatorname{Rad} \mathbb{A} G(X)= \begin{cases}2 & \text { If } X \text { has an isolated point. } \\ 3 & \text { If } X \text { does not have any isolated point. }\end{cases}
$$

Proof. It concludes from [6, Corollary 5.4], Theorem 4.1 and Corollary 4.2.

Theorem 4.5. The following statements are equivalent.

(a) The zero ideal of $R$ is an anti fixed-place ideal.

(b) $\Gamma(R)$ is triangulated.

(c) $\operatorname{Min}(R)$ does not have any isolated point.

Proof. (a) $\Rightarrow$ (b). Suppose that $\Gamma(R)$ is not triangulated, then $a \in Z(R)^{*}$ exists such that $a$ is not a vertex of any triangle, so, by Lemma 3.6, we have $h_{m}(a)$ is singleton, hence there is a $P \in \operatorname{Min}(R)$ such that $h_{m}(a)=\{P\}$. Since $h_{m}(a)$ is open and $\left\{h_{m}^{c}(x): x \in R\right\}$ is a base for $Y$, it follows that $b \in R$ exists such that $P \in h_{m}^{c}(b) \subseteq h_{m}(a)=\{P\}$, thus $h_{m}^{c}(b)=\{P\}$, and, therefore $\operatorname{Ann}(b)=P$. It shows that $P$ is an affiliated prime ideal, hence $P \in \mathcal{B}(R) \neq \emptyset$ and consequently the zero ideal is not an anti fixed-place ideal.

(b) $\Rightarrow$ (c). By [29, Theorem 3.1], the space $\operatorname{Spec}(R)$ does not have any quasi-isolated point, i.e., $\operatorname{Min}(R)$ does not have any isolated point.

(c) $\Rightarrow$ (a). It concludes from [6, Corollary 4.3].

Theorem 4.6. The following statements are equivalent.

(a) The zero ideal of $R$ is an anti fixed-place ideal.

(b) $\mathbb{A} \mathbb{G}(R)$ is triangulated.

(c) $\operatorname{Min}(R)$ does not have any isolated point. 
Proof. (a) $\Rightarrow(\mathrm{b})$. It is similar to the proof of part (a) $\Rightarrow$ (b) of the previous theorem.

(b) $\Rightarrow$ (a). Suppose that the zero ideal of $R$ is not an anti fixed-place ideal. Then $P \in \mathcal{B}(R) \neq \emptyset$ exists, hence $P$ is an affiliated prime ideal, so there is some $a \in R$ such that $\operatorname{Ann}(a)=P$ and thus $h_{m}^{c}(a)=\{P\}$. This implies that $\{P\}$ is open in $\operatorname{Min}(R)$, therefore $h_{m}^{c}(P)=\operatorname{Min}(R) \backslash\{P\}$ is closed and consequently $\overline{h_{m}^{c}(P)}=\operatorname{Min}(R) \backslash\{P\}$. Thus $h_{m}(P)^{\circ}=\left(\overline{h_{m}^{c}(P)}\right)^{c}=\{P\}$. Now Lemma 3.6 concludes that $P$ is not a vertex of any triangle, and, therefore $\mathbb{A} \mathbb{G}(R)$ is not triangulated.

(a) $\Leftrightarrow(\mathrm{c})$. It is clear, by [6, Corollary 4.3$]$.

In [29, Corollary 3.3], it is asserted that "Let $R$ be a reduced ring and let $\operatorname{Spec}(R)$ be finite. Then $\Gamma(R)$ is a triangulated graph if and only if $\operatorname{Spec}(R)$ has no isolated points.". If $\operatorname{Spec}(R)$ is finite, then $\operatorname{Min}(R)$ is finite, so the zero ideal of $R$ is fixed-place, and, therefore it is not anti fixed-place. Hence, by the above theorem, $\Gamma(R)$ is not triangulated. Hence the assumption " $\Gamma(R)$ is a triangulated graph" in this assertion is not possible.

Now we can conclude the following corollary from the above theorems.

Corollary 4.7. $\Gamma(R)$ is triangulated if and only if $\mathbb{A} \mathbb{G}(R)$ is triangulated.

Now we can conclude easily from Theorem 4.5 and [6, Corollary 5.4] that $\Gamma(X)$ is triangulated if and only if $X$ does not have any isolated point. This fact is shown in [15, Proposition 2.1]. Also, we can conclude easily from Theorem 4.6 and $[6$, Corollary 5.4] that $\mathbb{A} \mathbb{G}(X)$ is triangulated if and only if $X$ does not have any isolated point. This fact also is shown in $[16$, Theorem 4.5].

If $\operatorname{Min}(R)$ is finite, then the zero ideal of $R$ is fixed-place, hence it is not anti fixed-place, and, therefore $\operatorname{Rad} \Gamma(R)=\operatorname{Rad} \mathbb{A} G(R)=2$, , by Corollary 4.2.

Suppose that $D$ is an integral domain and $R$ be an arbitrary ring. Then $\{0\} \times R \in \mathcal{B}(D \times R) \neq \emptyset$, so the zero ideal of $D \times R$ is not an anti fixedplace ideal, thus, by Corollary 4.2 and Theorems 4.5 and $4.6, \operatorname{Rad} \Gamma(R)=$ $\operatorname{Rad} \mathbb{A} G(R)=2$ and the graphs $\mathbb{A} \mathbb{G}(R)$ and $\Gamma(R)$ are not triangulated.

\section{Domination Number}

The main purpose of this section is studying of domination numbers of $\mathbb{A} \mathbb{G}(R)$ and then $\mathbb{A} \mathbb{G}(X)$. In this studying, we employ the Bourbaki associated prime divisor of the zero ideal and the fixed-place ideal notions.

Lemma 5.1. Let I be an ideal in $\mathbb{A}(R)^{*}$. The following statements are equivalent. 
(a) I is prime.

(b) $I$ is a maximal element of $\mathbb{A}(R)^{*}$.

(c) I is a Bourbaki associated prime divisor of the zero ideal of $R$.

Proof. (a) $\Rightarrow(\mathrm{b})$. Suppose that $I \subseteq J$ and $J \in \mathbb{A}(R)^{*}$, then $0 \neq a \in \operatorname{Ann}(J)$ exists. Since $R$ is a reduced ring, it follows that $a \notin J$, then $a \notin I$ and $a J \subseteq I$, hence $J \subseteq I$ and thus $I=J$. Consequently, $I$ is a maximal element of $\mathbb{A}(R)^{*}$.

(b) $\Rightarrow(\mathrm{c})$. Since $I \in \mathbb{A}(R)^{*}$, it follows that there is some $0 \neq a \in R$ such that $\operatorname{Ann}(a)=I$. Suppose that $x y \in I$ and $x \notin I$, then $I=\operatorname{Ann}(a) \subseteq$ $\operatorname{Ann}(a x)$, so $y \in \operatorname{Ann}(a x) \subseteq \operatorname{Ann}(a)=I$, by the maximality of $I$, hence $I$ is prime, and, therefore $I$ is a Bourbaki associated prime divisor of the zero ideal.

(c) $\Rightarrow(\mathrm{a})$. It is clear.

Proposition 5.2. The following statements hold.

(a) Suppose that $I \in \mathbb{A}(R)^{*} . I$ is contained in some maximal element of $\mathbb{A}(R)^{*}$ if and only if $\operatorname{Min}(I) \cap \mathcal{B}(R) \neq \emptyset$.

(b) Every element of $\mathbb{A}(R)^{*}$ is contained in some maximal element of $\mathbb{A}(R)^{*}$ if and only if the zero ideal of $R$ is a fixed-place ideal.

(c) $\mathbb{A}(R)^{*}$ does not have any maximal element if and only if the zero ideal of $R$ is an anti fixed-place ideal.

Proof. $(\mathrm{a} \Rightarrow)$. By Lemma 5.1, some $P \in \mathcal{B}(R)$ exists such that $I \subseteq P$, since $P \in \operatorname{Min}(R)$, it follows that $P \in \operatorname{Min}(I)$, and, therefore $P \in \mathcal{B}(R) \cap \operatorname{Min}(R) \neq$ $\emptyset$.

( $\mathrm{a} \Leftarrow)$. It is clear, by Lemma 5.1.

$(\mathrm{b} \Rightarrow)$. On the contrary, suppose that $\bigcap_{P \in \mathcal{B}(R)} P \neq\{0\}$ and thus there is some $0 \neq a \in \bigcap_{P \in \mathcal{B}(R)} P$. Then

$$
\operatorname{Ann}(a)=(0: a)=\left(\bigcap_{P \in \operatorname{Min}(R)} P: a\right)=\bigcap_{a \notin P \in \operatorname{Min}(R)} P
$$

By the assumption, there is some $P_{\circ} \in \mathcal{B}(R)$ such that $\operatorname{Ann}(a) \subseteq P_{\circ}$, then $\bigcap_{a \notin P \in \operatorname{Min}(R)} P \subseteq P_{\circ}$, and, therefore

$$
\bigcap_{P_{\circ} \neq P \in \operatorname{Min}(R)} P \subseteq \bigcap_{a \notin P \in \operatorname{Min}(R)} P \subseteq P_{\circ}
$$




$$
\Rightarrow\{0\}=\left(\bigcap_{P_{\circ} \neq P \in \operatorname{Min}(R)} P\right) \cap P_{\circ}=\bigcap_{P_{\circ} \neq P \in \operatorname{Min}(R)} P
$$

which is a contradiction.

$(\mathrm{b} \Leftarrow)$. By the assumption, $\bigcap_{P \in \mathcal{B}(R)} P=\{0\}$. So

$$
\operatorname{Ann}(I)=(0: I)=\left(\bigcap_{P \in \mathcal{B}(R)} P: I\right)=\bigcap_{P \in \mathcal{B}(R)}(P: I)=\bigcap_{I \nsubseteq P \in \mathcal{B}(R)} P
$$

Hence $P \in \mathcal{B}(R)$ exists such that $I \subseteq P$ and thus, by Lemma 5.1. It completes the proof.

(c). It is evident, by Lemma 5.1 .

In the proof of [27, Theorem 2.2], it is claimed that "By Zorn's Lemma, it is clear that if $\mathbb{A}(R)^{*} \neq \emptyset$, then $\mathbb{A}(R)^{*}$ has a maximal element" and "for every $J \in \mathbb{A}(R)^{*}$, there exists $I \in M$ [the family of all maximal elements of $\mathbb{A}(R)^{*}$ ] such that $J \subseteq I^{\prime \prime}$. But the chain of elements of $\mathbb{A}(R)^{*}$ need not have an upper bound and thus $\mathbb{A}(R)^{*}$ does not satisfy the conditions of Zorn's Lemma. By the above proposition, an ideal of $\mathbb{A}(R)^{*}$ need not contained in a maximal element of $\mathbb{A}(R)^{*}$. In the following example, we give a counterexample.

Example 5.3. Since $\mathbb{R}$ does not have any isolated point, by [6, Corollary 5.4], the zero ideal of $C(\mathbb{R})$ is an anti fixed-place ideal and therefore $\mathcal{B}(C(\mathbb{R}))=\emptyset$. Then Lemma 5.1 implies that the family of all maximal elements of $\mathbb{A}(C(\mathbb{R}))^{*}$ is empty.

Also, in [27, Theorem 2.2], it is asserted that "Let $M$ be the set of all maximal elements of the set $\mathbb{A}(R)^{*}$. If $|M|>1$, then $\operatorname{dt}_{t} \mathbb{A} \mathbb{G}(R)=M^{\prime}$. In Example 5.8, we show that this assertion is not true.

In the following theorem, we show that the cardinal of $\mathcal{B}(R)$ is a lower bound of $\mathrm{dt}_{t}(\mathbb{A} \mathbb{G}(R))$. Also, Theorem 5.5 shows that if the zero ideal of $R$ is fixed-place, then $|\mathcal{B}(R)|=\mathrm{dt}_{t}(\mathbb{A} \mathbb{G}(R))$. Finally, we know that if $\operatorname{Min}(R)$ is a finite set (for example $R$ is a Noetherian ring), then the zero ideal of $R$ is a fixed-place ideal and thus $|\operatorname{Min}(R)|=|\mathcal{B}(R)|=\mathrm{dt}_{t}(R)$.

Theorem 5.4. The following hold.

(a) $|\mathcal{B}(R)| \leqslant \mathrm{dt}_{t}(\mathbb{A} \mathbb{G}(R))$.

(b) If $|\operatorname{Min}(R)|>2$, then $|\mathcal{B}(R)| \leqslant \operatorname{dt}(\mathbb{A} \mathbb{G}(R))$.

Proof. (a). Suppose that $D$ is a total dominating set of $\mathbb{A} \mathbb{G}(R)$. For each $P \in \mathcal{B}(R)$, there is some $I_{P} \in D$, such that $I_{P}$ is adjacent to $P$, so $P I_{P}=\{0\}$, hence $P \subseteq \operatorname{Ann}\left(I_{P}\right)$ and thus $P=\operatorname{Ann}\left(I_{P}\right)$, by Lemma 5.1. Now suppose 
that $I_{P}=I_{Q}$, for some $P, Q \in \mathcal{B}(R)$, then $P=\operatorname{Ann}\left(I_{P}\right)=\operatorname{Ann}\left(I_{Q}\right)=Q$ and thus the map $P \rightsquigarrow I_{P}$ is one-to-one. This implies that $|\mathcal{B}(R)| \leqslant|D|$ and consequently $|\mathcal{B}(R)| \leqslant \mathrm{dt}_{t}(\mathbb{A} \mathbb{G}(R))$.

(b). Let $D$ be a dominating set. For each $P \in \mathcal{B}(R)$, if $P \in D$, then we set $K_{P}=P$ and if $P \notin D$, there is some $K_{P} \in D$ such that $K_{P}$ is adjacent to $P$. Suppose that $K_{P}=K_{Q}$, for some $P, Q \in \mathcal{B}(R)$. If $P, Q \in D$, then $P=K_{P}=K_{Q}=Q$. If $P, Q \notin D$, then $P$ and $Q$ are adjacent to $K_{P}$ and $K_{Q}$, respectively, so $P K_{P}=Q K_{Q}=\{0\}$, thus $P \subseteq \operatorname{Ann}\left(K_{P}\right)$ and $Q \subseteq \operatorname{Ann}\left(K_{Q}\right)$, and, therefore $P=\operatorname{Ann}\left(K_{P}\right)=\operatorname{Ann}\left(K_{Q}\right)=Q$, by Lemma 5.1. Finally, without loss of generality, we assume $P \in D$ and $Q \notin D$, then $P=K_{P}$ and $K_{Q}$ is adjacent to $Q$, so $P$ is adjacent to $Q$ and thus $P Q=\{0\}$. Hence for each $P^{\prime} \in \operatorname{Min}(R)$, we have $P Q=\{0\} \subseteq P^{\prime}$, and, therefore either $P \subseteq P^{\prime}$ or $Q \subseteq P^{\prime}$, and, therefore, by Lemma 5.1, either $P=P^{\prime}$ or $Q=P^{\prime}$. This implies that $|\operatorname{Min}(R)| \leqslant 2$, which contradicts the assumption. Consequently, the map $P \rightsquigarrow K_{P}$ is one-to-one. This implies that $|\mathcal{B}(R)| \leqslant|D|$ and thus $|\mathcal{B}(R)| \leqslant \operatorname{dt}(\mathbb{A} \mathbb{G}(R))$.

Theorem 5.5. If the zero ideal of $R$ is a fixed-place ideal, then

(a) $\mathrm{dt}_{t}(\mathbb{A} \mathbb{G}(R))=|\mathcal{B}(R)|$.

(b) If $|\operatorname{Min}(R)|>2$, then $\operatorname{dt}(\mathbb{A} \mathbb{G}(R))=|\mathcal{B}(R)|$.

Proof. (a). By the above theorem, it is sufficient to show that $\mathrm{dt}_{t}(\mathbb{A} \mathbb{G}(R)) \leqslant$ $|\mathcal{B}(R)|$. For every $P \in \mathcal{B}(R)$, pick $a_{P} \in R$, such that $\operatorname{Ann}\left(a_{P}\right)=P$. For each $K \in \mathbb{A}(R)^{*}$, by the assumption and Proposition 5.2, there is some $P \in$ $\mathcal{B}(R)$ such that $K \subseteq P=\operatorname{Ann}\left(a_{P}\right)$, so $R a_{P} K=\{0\}$, and, therefore $K$ is adjacent to $R a_{P}$. This implies that $\left\{R a_{P}: P \in \mathcal{B}(R)\right\}$ is a dominating set and consequently $\mathrm{dt}_{t}(\mathbb{A} \mathbb{G}(R)) \leqslant|\mathcal{B}(R)|$.

(b). By the fact that $\operatorname{dt}(\mathbb{A} G(R)) \leqslant d_{t}(\mathbb{A G}(R))$, it follows from part (a) and the above theorem.

We recall that if $\operatorname{Min}(R)$ is finite, then the zero ideal of $R$ is a fixed-place ideal and $\operatorname{Min}(R)=\mathcal{B}(R)$. Thus [26, Theorem 2.4] and [27, Theorem 2.4] are immediate consequences of the above theorem. Also, we can conclude the following corollary from the above theorem and [6, Theorems 5.2 and 5.5].

Corollary 5.6. Suppose that $X$ is an almost discrete space. Then

(a) $\mathrm{dt}_{t}(\mathbb{A G}(X))=|I(X)|$.

(b) If $|X|>2$, then $\operatorname{dt}(\mathbb{A} \mathbb{G}(X))=|I(X)|$.

Theorem 5.7. If the zero ideal of a ring $R$ is not a fixed-place ideal, then $\operatorname{dt}(\mathbb{A} G(R))$ and $\operatorname{dt}_{t}(\mathbb{A} \mathbb{G}(R))$ are infinite. 
Proof. Suppose that $D$ is a dominating set of $\mathbb{A} \mathbb{G}(R)$. By Proposition 5.2, there is some $J_{1} \in \mathbb{A}(R)^{*}$ which is not contained in a maximal element of $\mathbb{A}(R)^{*}$. If $J_{1} \in D$, then we set $I_{1}=K_{1}=J_{1}$. If $J_{1} \notin D$, then there is some vertex $I_{1} \in D$ which is adjacent to $J_{1}$, then $J_{1} I_{1}=\{0\}$, so $J_{1} \subseteq \operatorname{Ann}\left(I_{1}\right)$. In this case, we set $K_{1}=\operatorname{Ann}\left(I_{1}\right)$. Since $J_{1}$ is not contained in a maximal element of $\mathbb{A}(R)^{*}$ and $J_{1} \subseteq K_{1}$, there is some $J_{2} \in \mathbb{A}(R)^{*}$ such that $K_{1} \subset J_{2}$. Similarly, we can find $K_{2} \in \mathbb{A}(R)^{*}$ in which either $I_{2}=K_{2} \in D$ or $K_{2}=\operatorname{Ann}\left(I_{2}\right)$, for some $I_{2} \in D$. By induction, we have the following chain

$$
J_{1} \subseteq K_{1} \subset J_{2} \subseteq K_{2} \subset \ldots \subset J_{n} \subseteq K_{n} \subset \ldots
$$

Now suppose that $n \neq m$, then $K_{n} \neq K_{m}$. Without loss of generality, we assume $n<m$, hence we have four cases

case 1: If $I_{n}=K_{n}$ and $I_{m}=K_{m}$, then it is evident that $I_{n} \neq I_{m}$.

case 2: If $K_{n}=\operatorname{Ann}\left(I_{n}\right)$ and $K_{m}=\operatorname{Ann}\left(I_{m}\right)$, so it is clear that $I_{n} \neq I_{m}$.

case 3: If $K_{n}=I_{n}$ and $K_{m}=\operatorname{Ann}\left(I_{m}\right)$, then $I_{n} \subset \operatorname{Ann}\left(I_{m}\right)$, so we have $I_{n} I_{m}=$ $\{0\}$. Hence $I_{n} \neq I_{m}$, because otherwise, $I_{n}^{2}=\{0\}$ and therefore $I_{n}=$ $\{0\}$, which is a contradiction.

case 4: If $K_{n}=\operatorname{Ann}\left(I_{n}\right)$ and $K_{m}=I_{m}$, then $\operatorname{Ann}\left(I_{n}\right) \subset I_{m}$, so we have $\operatorname{Ann}\left(I_{m}\right) \subseteq \operatorname{Ann}\left(\operatorname{Ann}\left(I_{n}\right)\right)$. Hence $I_{n} \neq I_{m}$, because otherwise, similar to case $3, \operatorname{Ann}\left(I_{n}\right)=\{0\}$, which is a contradiction.

Since $\left\{I_{n}: n \in \mathbb{N}\right\} \subseteq D$, it follows that $D$ is infinite and consequently $\operatorname{dt}(\mathbb{A} \mathbb{G}(R))$ is infinite. Hence $\operatorname{dt}_{t}(\mathbb{A} \mathbb{G}(R))$ is infinite, by the fact that

$$
\operatorname{dt}(\mathbb{A} \mathbb{G}(R)) \leqslant \operatorname{dt}_{t}(\mathbb{A} \mathbb{G}(R)) .
$$

Now by the above theorem, $\operatorname{dt}_{t}(\mathbb{A} \mathbb{G}(C(\mathbb{R})))$ and $\operatorname{dt}(\mathbb{A} \mathbb{G}(C(\mathbb{R})))$ are infinite, so the inequality in Theorem 5.4, can be proper.

Example 5.8. Suppose that $R=C(\mathbb{R}) \times \mathbb{R} \times \mathbb{R}$. It is easy to see that if $M$ is the family of all maximal elements of $\mathbb{A}(R)^{*}$, then $M=\mathcal{B}(C(\mathbb{R}) \times \mathbb{R} \times \mathbb{R})=$ $\{C(\mathbb{R}) \times \mathbb{R} \times\{0\}, C(\mathbb{R}) \times\{0\} \times \mathbb{R}\}$, by Lemma 5.1, Proposition 5.2 and Example 5.3. Since the zero ideal of $R$ is not a fixed-place ideal, by the above theorem, it follows that $\mathrm{dt}_{t}(R)$ is infinite. Thus $|M| \neq \mathrm{dt}_{t}(R)$.

Corollary 5.9. The following statements are equivalent

(a) $\operatorname{dt}_{t}(\mathbb{A} \mathbb{G}(R))$ is finite

(b) $\mathrm{dt}_{t}(\mathbb{A G}(R))$ is finite 
(c) $\operatorname{Min}(R)$ is finite

Proof. It follows immediately from Theorems 5.5 and 5.7 and the fact that if $\operatorname{Min}(R)$ is finite, then the zero ideal is a fixed-place ideal.

Finally in the following proposition, we generalize [27, Theorem 2.3] to the infinite version.

Proposition 5.10. For each reduced ring $R$, we have

$$
\mathrm{dt}_{t}(\Gamma(R)) \leqslant \mathrm{dt}_{t}(\mathbb{A} \mathbb{G}(R)) .
$$

Proof. Suppose that $D$ is a total dominating set of $\mathbb{A} \mathbb{G}(R)$. So for each $I \in P$, there is some $0 \neq a_{I} \in I$. For every $a \in R$, there is some $I \in D$ such that $I$ is adjacent to $R a$ in $\mathbb{A G}(R)$, thus $R a I=\{0\}$, hence $a a_{I}=0$, and, therefore $a_{I}$ is adjacent to $a$ in $\Gamma(R)$. Consequently, $\left\{a_{I}: I \in D\right\}$ is a total dominating set of $\Gamma(R)$ and this implies that $\mathrm{dt}_{t}(\Gamma(R)) \leqslant \mathrm{dt}_{t}(\mathbb{A} \mathbb{G}(R))$.

\section{References}

[1] Aalipour Gh, Akbari S, Behboodi M, Nikandish R, Nikmehr M J, Shaveisi F. The classification of the annihilating-ideal graphs of commutative rings. Algebra Colloq., 21(02):249-256, 2014. doi:10.1142/S1005386714000200

[2] Aalipour Gh, Akbari S, Nikandish R, Nikmehr M J, Shaveisi F. On the coloring of the annihilating-ideal graph of a commutative ring. Discrete Math., 312(17):2620-2626, 2012. doi:10.1016/j.disc.2011.10.020

[3] Afkhami M, Barati Z, Khashyarmanesh K. A graph associated to a lattice. Ric. Mat., 63(1):67-78, 2014. doi:10.1007/s11587-013-0164-6

[4] Akbari S, Maimani H R, Yassemi S. When a zero-divisor graph is planar or a complete r-partite graph. Journal of Algebra, 270(1):169-180, 2003. doi:10.1016/S0021-8693(03)00370-3

[5] Akbari S, Mohammadian A. On the zero-divisor graph of a commutative ring. J. Algebra, 274(2):847-855, 2004. doi:10.1016/S0021-8693(03)004356

[6] Aliabad A R, Badie M. Fixed-place ideals in commutative rings. Comment. Math. Univ. Carolin., 54(1):53-68, 2013.

[7] Aliabad A R, Badie M. On Bourbaki associated prime divisors of an ideal. Quaest. Math., pages 1-22, 2018. doi:10.2989/16073606.2018.1459924 
[8] Aliabad A R, Badie M, Nazari S. An extension of $z$-ideals and $z^{\circ}$-ideals. Hacet. J. Math. Stat., 49(1):254-272 2020. doi:10.15672/hujms.455030

[9] Aliabad A R and Mohamadian R. On $s z^{\circ}$-ideals in polynomial rings. Comm. Algebra, 39(2):701-717, 2011. doi:10.1080/00927871003591884

[10] Aliniaeifard F, Behboodi M. Rings whose annihilating-ideal graphs have positive genus. J. Algebra Appl., 11(03):1250049, 2012. doi: 10.1142/S021 9498811005774

[11] Anderson D F, Levy R, Shapiro R. Zero-divisor graphs, von neumann regular rings, and boolean algebras. J. Pure App. Algebra, 180(3):221241, 2003. doi:10.1016/S0022-4049(02)00250-5

[12] Anderson D F, Livingston P S. The zero-divisor graph of a commutative ring. J. Algebra, 217(2):434-447, 1999. doi:10.1006/jabr.1998.7840

[13] Assari A, Rahimi M. Graphs generated by measures. J. of Math., 2016, 2016. doi: $10.1155 / 2016 / 1706812$

[14] Atiyah M F, Macdonald I G. Introduction to commutative algebra, volume 2. Addison-Wesley Reading, MA, 1969.

[15] Azarpanah F and Motamedi M. Zero-divisor graph of $C(X)$. Acta Math. Hungar., 108(1-2):25-36, 2005. doi:10.1007/s10474-005-0205-Z

[16] Badie M. Annihilating-ideal graph of $C(X)$. J. Algebr. Syst., to apear.

[17] Beck I. Coloring of commutative rings. J. Algebra, 116(1):208-226, 1988. doi:10.1016/0021-8693(88)90202-5

[18] Behboodi M, Rakeei Z. The annihilating-ideal graph of commutative rings I. J. Algebra Appl., 10(04):727-739, 2011. doi:10.1142/S02194988110048 96

[19] Behboodi M, Rakeei Z. The annihilating-ideal graph of commutative rings II. J. Algebra Appl., 10(04):741-753, 2011. doi:10.1142/S02194988110049 02

[20] Bondy J A, Murty U S R. Graph theory with Application. The Macmillan Press, New York, 1976.

[21] Chelvam T, Selvakumar K. On the connectivity of the annihilatingideal graphs. Discuss. Math. Gen. Algebra Appl., 35(2):195-204, 2015. doi:10.7151/dmgaa.1241 
[22] Gillman L, Jerison M. Rings of continuous functions. Van. Nostrand Reinhold, New York, 1960.

[23] Henriksen M, Jerison M. The space of minimal prime ideals of a commutative ring. Trans. Amer. Math. Soc., 115:110-130, 1965. doi:10.2307/19942 60

[24] Levy R, Shapiro J. The zero-divisor graph of von neumann regular rings. 2002. doi:10.1081/AGB-120013178

[25] Mason G. Prime ideals and quotient rings of reduced rings. Sci. Math. Jpn., 34(6):941-956, 1989.

[26] Nikandish R, Maimani H R. Dominating sets of the annihilatingideal graphs. Electron. Notes Discrete Math., 45:17-22, 2014. doi:10.10 16/j.endm.2013.11.005

[27] Nikandish R, Maimani H R, Kiani S. Domination number in the annihilating-ideal graphs of commutative rings. Publ. Inst. Math., 97(111): 225231, 2015. doi:10.2298/PIM140222001N

[28] Redmond S. Central sets and radii of the zero-divisor graphs of commutative rings. Comm. Algebra, 34(7):2389-2401, 2006. doi:10.1080/00 927870600649103

[29] Samei K. The zero-divisor graph of a reduced ring. J. Pure App. Algebra, 209(3):813-821, 2007. doi:10.1016/j.jpaa.2006.08.008

[30] Sharp R Y. Steps in commutative algebra, volume 51. Cambridge university press, 2000.

[31] Willard S. General Topology. Addison Wesley Publishing Company, New York, 1970.

Mehdi Badie,

Department of Mathematics,

Jundi-Shapur University of Technology,

Dezful, Iran.

Email: badie@jsu.ac.ir 